\title{
Transforming Carbon Black into Graphene Oxide Quantum Dots by Pulsed Laser Ablation in Ethanol
}

\author{
Jung-Il Lee and Jeong Ho Ryu* \\ Department of Materials Science and Engineering, Korea National University of Transportation, \\ Chungbuk 27469, Republic of Korea
}

\begin{abstract}
Graphene oxide quantum dots (GOQDs) are nanometer-sized graphene oxide fragments that exhibit unique properties, making them interesting candidates for a range of new applications. Carbon black, one of the commercially available carbon precursors, is produced by the thermal decomposition or incomplete combustion of organic compounds. It is commonly used as a supporting material for catalysts because of its excellent electrical conductivity, high surface area, and stability. In this paper, we report the transformation of carbon black into GOQDs in 10 min using a one-step facile approach. This transformation was achieved by pulsed laser ablation (PLA) in ethanol using the earth-abundant and low-cost carbon black as precursor. Only ethanol and carbon black were used for the transformation. The carbon clusters ablated from the carbon black were completely transformed into GOQDs with a homogeneous size distribution and heights in the range of 0.3-1.7 nm. This confirmed that the transformed GOQDs consisted of only single- or few-layered graphene quantum dots. The UV-vis spectra showed absorption bands at 215, 260, and $320 \mathrm{~nm}$, which were attributed to the $\pi \rightarrow \pi^{*}$ transition of the $\mathrm{C}=\mathrm{C}$ of the $\mathrm{sp}^{2} \mathrm{C}$ bond in the $\mathrm{sp}^{3} \mathrm{C}$ matrix. A distinct blue emission peak at $450 \mathrm{~nm}$ was evident at an excitation wavelength of $360 \mathrm{~nm}$. The broader PL emission spectra are due to the oxygen-related functional groups emitting PL between 300 and $440 \mathrm{~nm}$.
\end{abstract}

(Received September 8, 2020; Accepted September 23, 2020)

Keywords: graphene oxide quantum dots, pulsed laser ablation, carbon black

\section{INTRODUCTION}

Quantum dots are nanoparticles with sizes smaller than the exciton Bohr radius of their bulk counterparts [1-3]. The threedimensional confinement of excitons results in interesting quantum confinement effects [4-6]. Graphene oxide quantum dots (GOQDs), in particular, are of great interest because of their unique optical, electronic, thermal and mechanical properties, and are considered promising materials for use in nanoelectronics [7-9]. Unlike many semiconducting materials, GOQDs are single-atom-thick nanomaterials with an infinite exciton Bohr radius [10]. Their charge carriers have desirable intrinsic mobility, with zero effective mass, and can drift over micrometers without scattering at room temperature. The charge carriers can also support current densities that are six

- 이정일·류정호: 교수

*Corresponding Author: Jeong Ho Ryu

[Tel: +82-43-841-5384, E-mail: jhryu@ut.ac.kr]

Copyright (c) The Korean Institute of Metals and Materials orders of magnitude higher than those of copper [12].

To utilize their properties in applications, it is necessary to have an efficient and reliable way of preparing the GOQDs. Various GOQD preparation methods and techniques, including organic synthesis, hydrothermal cutting, electrothermal processing, electron-beam lithography, and the transformation of $\mathrm{C}_{60}$ molecules have been attempted [13-17]. However, these might not be practical under critical synthesis conditions. Some are time-consuming because they involve multiple synthesis steps. Moreover, wet-chemical cutting is usually performed under strong acidic or basic conditions, which requires expensive neutralization for an extended period. Therefore, a simple, fast, and facile preparation method is needed.

Pulsed laser ablation (PLA) in liquid is a unique technique that can be used to prepare nanomaterials in a short period of time $[18,19]$. PLA can create highly non-equilibrium high temperature and pressure conditions in a liquid medium, in which irregular reactions and ablated species growth can occur $[20,21]$. Carbon black is a commercial carbon precursor pro- 
duced by the thermal decomposition or incomplete combustion of organic compounds. It has a well-defined morphology and a minimum content of tars or other unnecessary materials. Carbon black is typically used as a supporting material for catalysts because of its excellent electrical conductivity, high surface area, and stability [22]. Carbon black is comprised of sub-nanometer-sized crystalline carbon domains with defects that are linked by aliphatic amorphous carbon [23]. These carbon black sub-nanoscale crystalline domains are small and difficult to control in electrical and optical applications [24].

In this paper, we report a fast, simple, facile and cost-effective method to transform carbon black, which is an inexpensive carbon source, into GOQDs. This single-step process does not need complex vacuum apparatus or additional chemicals. The resulting prepared GOQDs were approximately $10 \mathrm{~nm}$ in size, and most of them were monolayered. They also exhibited blue luminescence, which is highly desirable for practical applications.

\section{EXPERIMENTAL PROCEDURE}

Vulcan CX-72 carbon black (Cabot Corporation) was used as the carbon source to prepare the GOQDs. Fig. 1 shows a schematic diagram of the experimental setup. A solution prepared with $1.0 \mathrm{~g}$ of carbon black in $500 \mathrm{~mL}$ of ethanol (> $99 \%$, Sigma Aldrich) was ultrasonicated for $1 \mathrm{~h}$ to homogeneously disperse the carbon black. PLA was then performed on the resultant suspension for $10 \mathrm{~min}$ at room temperature in air using an Nd:YAG laser system. The laser wavelength, power density, energy, repetition rate, pulse, and maximum output were $355 \mathrm{~nm}, 3.0 \mathrm{~W} / \mathrm{cm}^{2}, 100 \mathrm{~mJ} /$ pulse, $10 \mathrm{~Hz}$, $10 \mathrm{~nm}$, and $2.0 \mathrm{~J}$, respectively, and it was focused on a spot on the liquid surface (diameter of $10 \mathrm{~mm}^{2}$ ).

High-resolution transmission electron microscopy (HRTEM) images were obtained using a JEM-2100F transmission electron microscope equipped with a field emission gun (USA $200 \mathrm{kV}$ ). X-ray photoelectron spectroscopy (XPS) profiles were recorded using a VG ESCALAB 220i system (Thermo Scientific, USA). The XPS survey and high-resolution scans were carried out at pass energies of 100 and $20 \mathrm{eV}$, respectively. The $\mathrm{X}$-ray beam size was approximately $100 \mu \mathrm{m}$. The GOQD samples for the XPS and atomic force microscopy (AFM) measurements were prepared on a silicon substrate by a spin-

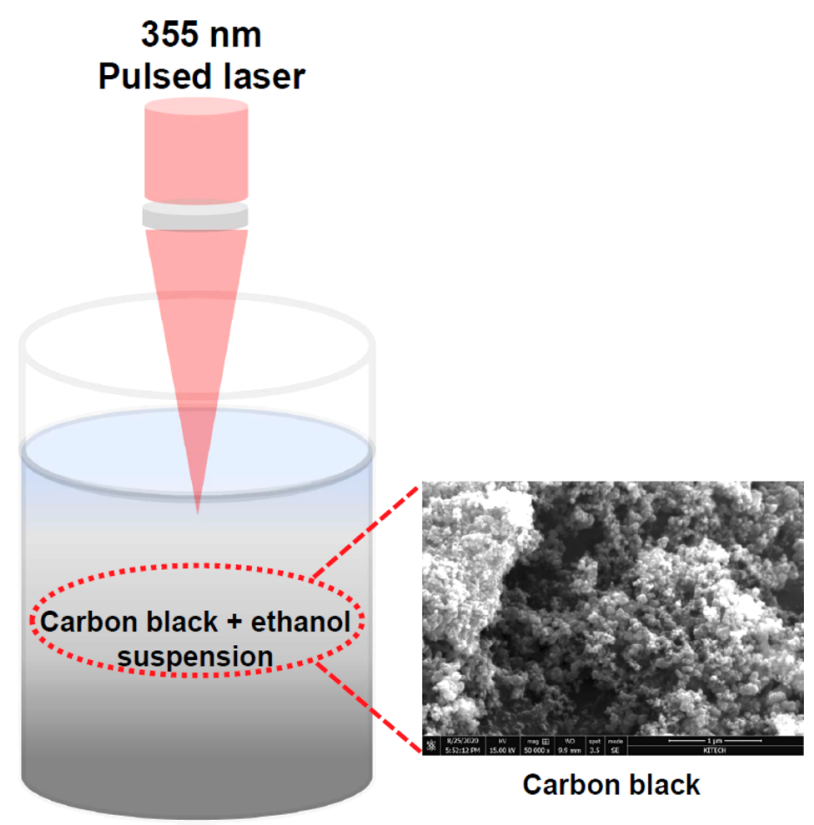

Fig. 1. Schematic diagram of PLA of the carbon black in the ethanol. The SEM image shows microstructure of the carbon black precursor.

coating at a rotation speed of $3000 \mathrm{rpm}$. The silicon substrate was dried at room temperature for $2 \mathrm{~h}$ before measurement. The photoluminescence (PL) spectra were obtained at room temperature using a PL spectrophotometer (Horiba, with a $150 \mathrm{~W}$ xenon arc lamp) in the wavelength range of $300 \mathrm{~nm}$ to $800 \mathrm{~nm}$. A time-correlated single photon counting (TCSPC) spectrometer (Horiba Jobin Yvon) was used to measure nanosecond lifetime. The ultraviolet-visible (UV-vis) absorption spectra were obtained using a Lambda $650 \mathrm{~S}$ UV-vis spectrophotometer (PerkinElmer).

\section{RESULTS AND DISCUSSION}

Fig. 2(a) shows the transmission electron micrographs of the carbon black particles transformed into GOQDs after PLA in ethanol for $10 \mathrm{~min}$. The distinct crystal structure of the transformed GOQDs was observed using a high-resolution transmission electron microscope (inset of Fig. 1(a)). A $0.24 \mathrm{~nm}$-long lattice fringe corresponding to the [1120] plane was clearly observed in the HR-TEM image. The insets on the right side of Fig. 1(a) show the edge structure of the GOQDs, which appears to be parallel to the zigzag orientation [25]. The formation of GOQDs with a typical edge structure is strongly dependent on how the $\mathrm{sp}^{3}$ carbon bond- 
ing structure is transformed into a sub-nanometer $\mathrm{sp}^{2}$ carbon bonding structure [26, 27]. No crystalline features corresponding to graphite, such as its [002] plane, were observed in HR-TEM [28]. Approximately 100 nanoparticles were used to determine the average crystal diameter. The size distribution of the transformed GOQD samples was fitted by a Gaussian distribution curve with a $95 \%$ confidence interval as shown in Fig. 1(b). An average diameter of $9.6 \mathrm{~nm}$ with a standard deviation (SD) of $3.5 \mathrm{~nm}$ was obtained. In addition, the height of the GOQD as determined by AFM was 0.3$1.7 \mathrm{~nm}$, which confirms that the transformed GOQDs consisted of only single or few-layered graphene quantum dots, as shown in Fig. 2(c) [29].

The possible mechanism for the transformation of carbon black into GOQDs via PLA in ethanol may be similar to that for coal $[28,30]$. A Coulomb explosion occurs at the surface of the carbon black particles during pulsed laser injections, after which a high-temperature and high-pressure plasma plume develops around the carbon black particles, due to multi-photon absorption [31]. Subsequently, a cavitation bubble is generated in the liquid as the plasma plume expands and cools. Carbon ablated from the carbon black particles clusters in the cavitation bubble and accumulates because of its high surface energy. This results in the formation of graphene layers as the temperature and internal pressure of the bubble decrease to below those of the surrounding liquid [32]. In this way, carbon clusters can be generated in the cavitation bubbles by pulsed laser irradiation on the carbon black particle surface. These carbon clusters are unstable because of their high surface energy and tend to aggregate [30]. Moreover, the carbon and hydroxy molecules produce a two-dimensional (2D) nanostructure because the hexagonal structure of carbon has a lower formation energy [33].

The chemical composition and functional groups on the transformed GOQD surface were determined from XPS measurements. In Fig. 3(a), the XPS results show a dominant graphitic $\mathrm{C} 1 \mathrm{~s}$ peak at $284.8 \mathrm{eV}$ and an $\mathrm{O} 1 \mathrm{~s}$ peak at $532.2 \mathrm{eV}$. The compositional ratio of $\mathrm{C} / \mathrm{O}$ observed in the transformed GOQDs was lower than that in carbon black, which is probably due to the bonding of the oxygen functional groups [34]. Fig. 3(b) shows the high-resolution C1s spectra of the transformed GOQDs, which are deconvoluted into three bands with binding energies of $284.4,286.6$ and $288.4 \mathrm{eV}$, asso-
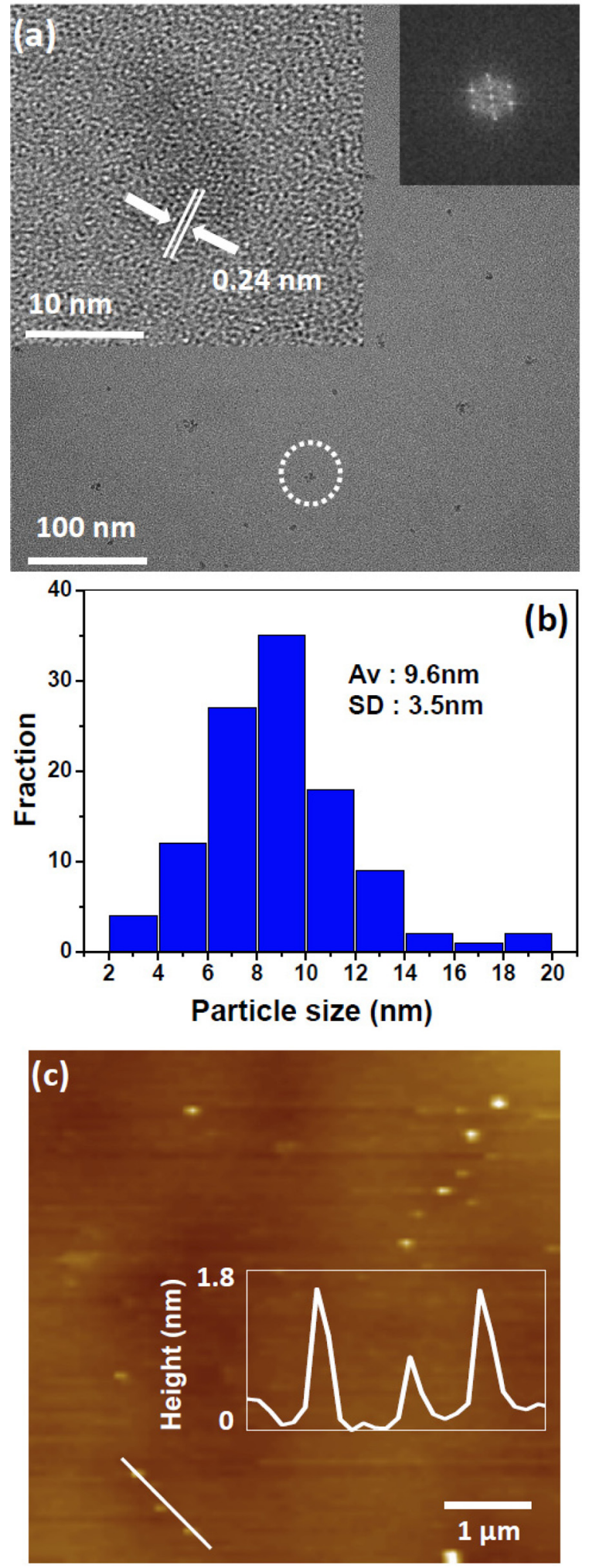

Fig. 2. TEM and AFM images of the transformed GOQDs. (a) TEM image of the GOQDs. Insets are the HR-TEM image (left) and 2D FFT pattern (right). (b) Size distribution of the GOQDs and HR-TEM image (inset). (c) AFM image and height profile of the GOQDs. 

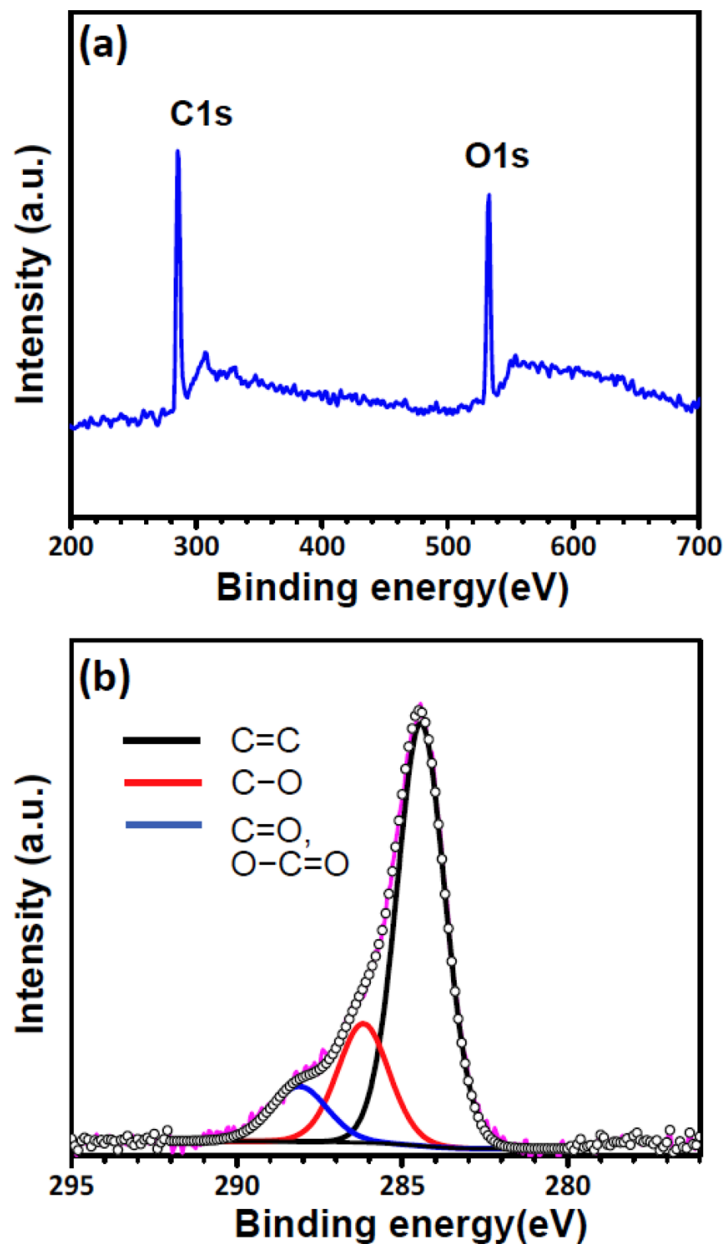

Fig. 3. Full survey XPS profile (a) and C1s XPS profile (b) of the transformed GOQDs.

ciated with the $\mathrm{C}=\mathrm{C}, \mathrm{C}-\mathrm{O}$, and $\mathrm{C}=\mathrm{O}$ and $\mathrm{COOH}$ bonds, respectively. Therefore, the transformed GOQDs contained hydroxyl, carbonyl, and carboxylic functional groups. In addition, more oxidation occurred in the GOQDs compared to carbon black, as confirmed by the decrease in the measured $\mathrm{sp}^{2}$ and $\mathrm{sp}^{3}$ ratios [35]. A higher fraction of oxygen functional groups facilitates the easy dispersion of the transformed GOQDs in ethanol, which is an essential property for practical applications, such as bioimaging.

We also investigated the optical properties of the transformed GOQDs by UV-Vis absorption and PL spectroscopies. The UV-Vis spectra showed characteristic absorption bands at 215, 260, and $320 \mathrm{~nm}$ as shown in Fig. 4(a). The absorption bands at 215 and $260 \mathrm{~nm}$ were due to the $\pi \rightarrow \pi^{*}$ transition of the $\mathrm{C}=\mathrm{C}$ of the $\mathrm{sp}^{2} \mathrm{C}$ bond in the $\mathrm{sp}^{3} \mathrm{C}$ matrix.
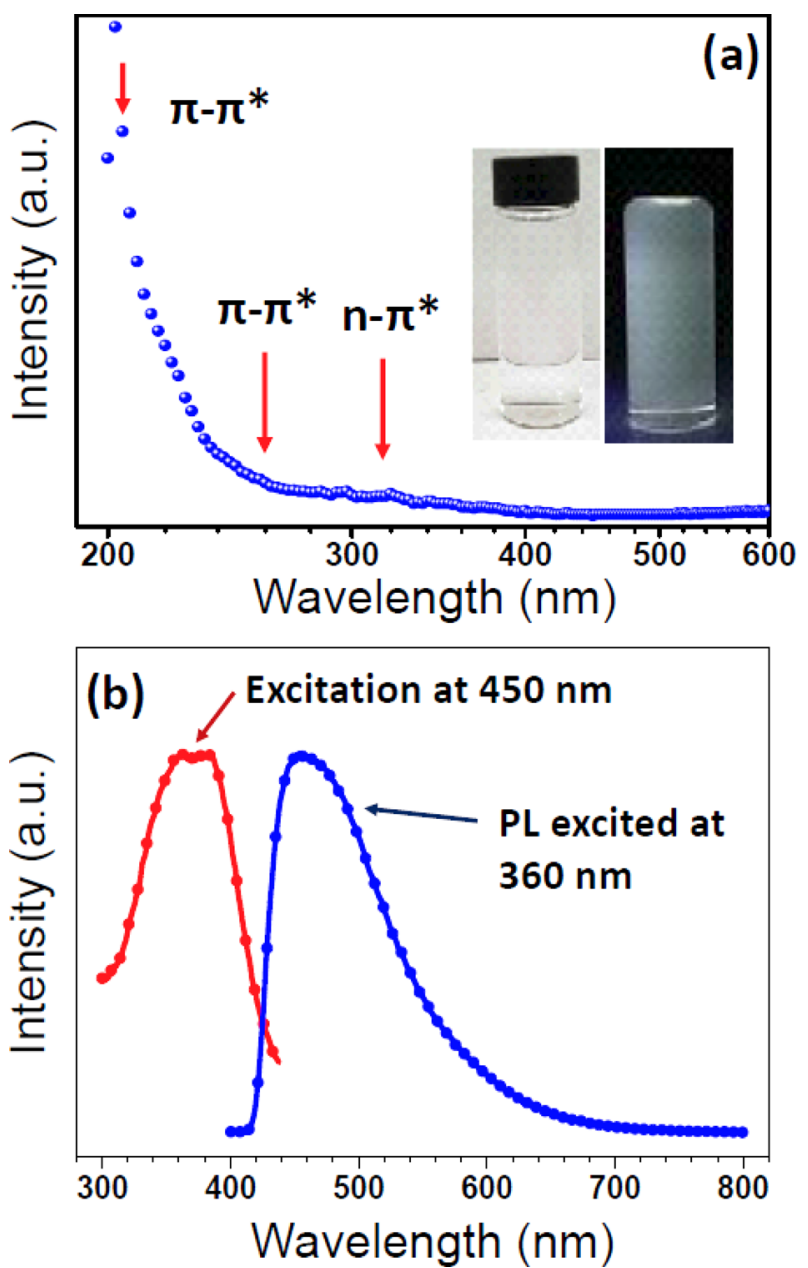

Fig. 4. UV-vis absorption (a) and PL spectrum (b). The x-axis of (a) is $\log _{10}$ scale. The excitation curve was recorded at $450 \mathrm{~nm}$ and emission spectrum were recorded at $360 \mathrm{~nm}$. Insets of (a) show the digital photographs under daylight (left) and $365 \mathrm{~nm}$ UV lamp (right) illumination.

Meanwhile, the shoulder peak around $320 \mathrm{~nm}$ is due to the $\mathrm{n}$ $\rightarrow \pi^{*}$ transition of the $\mathrm{C}=\mathrm{O}[3,36]$. The GOQDs transformed from carbon black are characterized by intense UV-Vis spectra at the $\pi \rightarrow \pi^{*}$ transition (215 and $260 \mathrm{~nm}$ ). This indicates a high density of zig-zag sites, which formed after laser irradiation. Qu and coworkers reported that in their oxygenreduction experiment, they observed no change in the UV-vis spectra around $320 \mathrm{~nm}$. Thus, this shoulder peak is related to the $\mathrm{n} \rightarrow \pi^{*}$ transition of the $\mathrm{C}=\mathrm{O}$ bond. [37].

Fig. 4(b) represents the PL excitation and emission spectra of the transformed GOQDs. The distinct blue emission peak at $450 \mathrm{~nm}$ is evident at an excitation wavelength of $360 \mathrm{~nm}$. The broader PL emission spectra are due to the oxygen- 
related functional groups emitting PL between 300 and $440 \mathrm{~nm}$. The insets in Fig. 4(b) show digital photographs of the prepared GOQD sample under an excitation wavelength of $360 \mathrm{~nm}$. The PL mechanism of the GOQDs can be attributed to defect-state emissions related to the oxygenrelated functional groups [38]. The transformed GOQDs have high concentrations of oxygen-related surface functional groups, as revealed by XPS (Fig. 3(b)). In addition, the observed PL property can be traced to the various defect sites of the surface oxygen functional groups.

The origin of the optical properties from GOQDs remains an important question and has been tentatively suggested to be related to emissive traps, aromatic structures, and the density of free zig-zag sites [25,36-38]. To investigate the recombination mechanism of the GOQDs, we performed timeresolved photoluminescence (TRPL) analysis as shown in Fig. 5(a). The fluorescence decay curve was fitted with a triexponential function (Eq. 1), where fluorescence decay occured through three different relaxation pathways [30].

$$
\text { Fit }=\mathrm{A}+\mathrm{B}_{1} \mathrm{e}^{(-\mathrm{t} / \tau 1)}+\mathrm{B}_{2} \mathrm{e}^{(-\mathrm{t} / \tau 2)}+\mathrm{B}_{3} \mathrm{e}^{(-\mathrm{t} / \tau 3)}
$$

where " $\tau$ " is the fluorescence lifetime and " $\mathrm{B}$ " represents the amplitude of the corresponding lifetime. The obtained chisquare $\left(\chi^{2}\right)$ ranges between 1.2 and 1.3. A $\chi^{2}$ value in the range of $1.0<\chi^{2}<1.4$ is generally assumed to be acceptable for fitting. Also, among the three lifetimes, one is associated with an intrinsic state, while the other two are due to the existence of oxygen-rich functional groups on the surface of the GOQDs [39, 40]. Fluorescence lifetimes of the GOQDs were recorded at $450 \mathrm{~nm}$, where the excitation wavelength of a diode laser was $370 \mathrm{~nm}$. The lifetimes of the transformed GOQDs were $\tau 1=0.8 \mathrm{~ns}(49 \%), \tau 2=3.2 \mathrm{~ns}(37 \%)$ and $\tau 3$ $=10 \mathrm{~ns}(13 \%)$. These lifetimes are similar to previously reported values [39, 40].

Fig. 5(b) summarizes the possible PL mechanism [39]. The GOQDs contain quite a number of disorder-induced defect states within the $\pi-\pi^{*}$ gap. The electrons of the GOQDs are excited to a higher energy level in the lowest unoccupied molecular orbital (LUMO) band. They then move to the defect-site states via a non-radiative relaxation process related to the PL properties of the GOQDs [40].

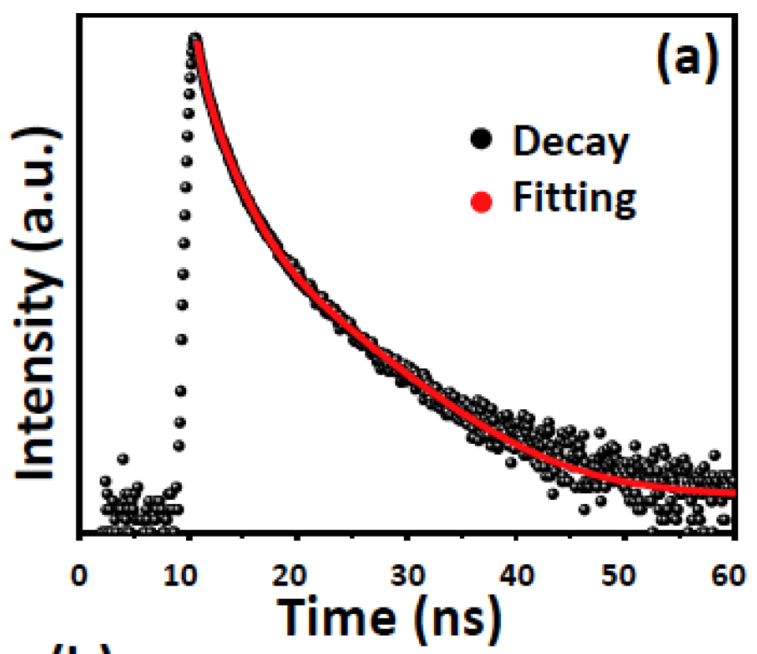

(b)

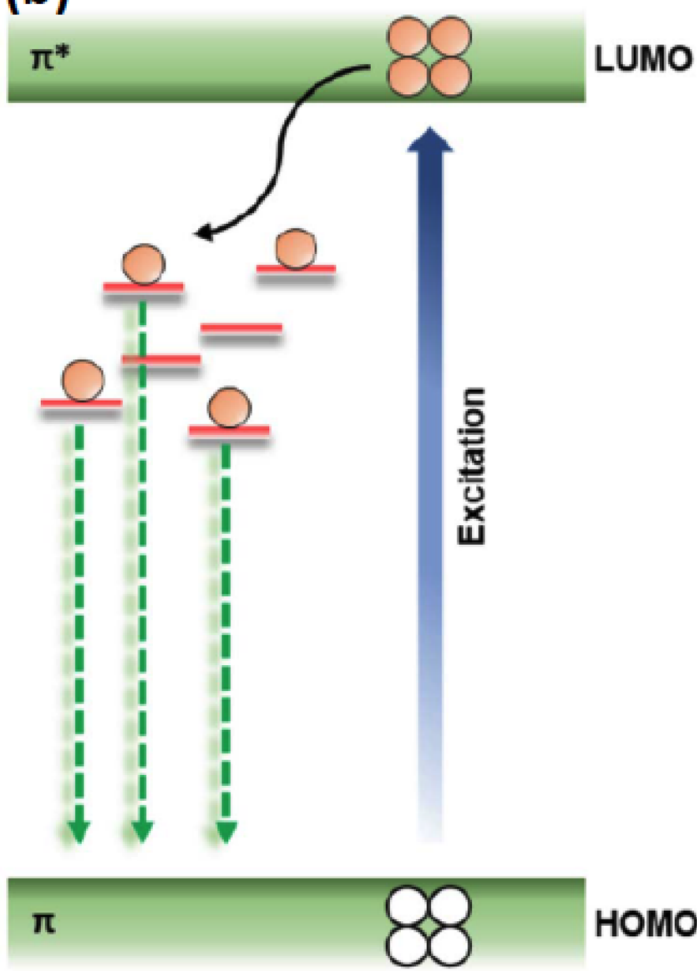

Fig. 5. Time-resolved photoluminescence (TRPL) spectrum (a) and schematic diagrams of the PL mechanism of the transformed GOQD (b).

\section{CONCLUSIONS}

We successfully transformed a carbon black precursor into GOQDs in less than 10 min by PLA in ethanol. The carbon clusters ablated from the carbon black were completely transformed into GOQDs with a homogeneous size distribution and heights in the range of $0.3-1.7 \mathrm{~nm}$. This confirmed that 
the transformed GOQDs consisted of only single- or few-layered graphene quantum dots. The UV-vis spectra showed absorption bands at 215, 260, and $320 \mathrm{~nm}$, which were attributed to the $\pi \mapsto \pi^{*}$ transition of the $\mathrm{C}=\mathrm{C}$ of the $\mathrm{sp}^{2} \mathrm{C}$ bond in the $\mathrm{sp}^{3} \mathrm{C}$ matrix. Meanwhile, the shoulder peak around $320 \mathrm{~nm}$ was attributed to the $\mathrm{n} \rightarrow \pi^{*}$ transition of the $\mathrm{C}=\mathrm{O}$ bond. The PL spectra exhibited a distinct blue emission peak at $450 \mathrm{~nm}$ under excitation at $360 \mathrm{~nm}$. The broader PL emission spectra might be due to the oxygen-related functional groups emitting PL spectra between 300 and $440 \mathrm{~nm}$.

\section{ACKNOWLEDGEMENT}

This research was supported by the Basic Science Research Program through the National Research Foundation of Korea (NRF) funded by the Ministry of Education (No. 2019R1I1 A3A0106266212).

\section{REFERENCES}

1. L. E. Brus, Appl. Phys. A 53, 465 (1991).

2. A. L. Efros and M. Rosen, Annu. Rev. Mater. Sci. 30, 475 (2000).

3. L. Li and S. Zhang, Chem. Commun. 48, 10177 (2012).

4. T. Takagahara and K. Takeda, Phys. Rev. B 46, 15578 (1992).

5. S. Zhu, Y. Song, J. Wang, H. Wan, Y. Zhang, Y. Ning, and B. Yang, Nano Today 13, 10 (2017).

6. T. Edvinsson, R. Soc. Open. Sci. 5, 180387 (2018).

7. J. Lu, P. S. E. Yeo, C. K. Gan, P. Wu, and K. P. Loh, Nat. Nanotechnol. 6, 247 (2011).

8. U.G. Hwang, Y.S. Lim, Korean J. Met. Mater. 57, 603 (2019).

9. T.-S. Jun, N.-H. Park, D.-S. So, J.-W. Lee, K. B. Shim, and H. Ham, J. Korean Cryst. Growth Cryst. Technol. 23, 325 (2013).

10. S. Zhu, J. Zhang, X. Liu, B. Li, X. Wang, S. Tang, Q. Meng, Y. Li, C. Shi, R. Hu, and B. Yang, RSC Adv. 2, 2717 (2012).

11. S. Z. Mortazavi, P. Parvin, and A. Reyhani, Laser Phys. Lett. 9, 547 (2012).

12. X. L. Li, X. R. Wang, L. Zhang, S. W. Lee, and H. J. Dai, Science 319, 1229 (2008).

13. D. Pan, J. Zhang, Z. Li, and M. Wu, Adv. Mater. 22, 734 (2010).

14. I.-J. Shon, J.-K. Yoon, K.-T. Hong, Met. Mater. Int. 24, 130
(2018).

15. R. Liu, D. Wu, X. Feng, and K. Müllen, J. Am. Chem. Soc. 133, 15221 (2011).

16. T. Gokus, R. R. Nair, A. Bonetti, M. Böhmler, A. Lombardo, K. S. Novoselov, A. K. Geim, A. C. Ferrari, and A. Hartschuh, ACS Nano 3, 3963 (2009).

17. G. Eda, Y. Y. Lin, C. Mattevi, H. Yamaguchi, H. A. Chen, I. S. Chen, C. W. Chen, and M. Chhowalla, Adv. Mater. 22, 505 (2010).

18. S. K. Kang, S. Mhin, H. Han, K. M. Kim, J. L. Jones, J. H. Ryu, J. S. Kang, S. H. Kim, and K. B. Shim, Sci. Rep. 6, 38423 (2016).

19. P. Russo, A. Hu, G. Compagnini, W. W. Duley, and N. Y. Zhou, Nanoscale 6, 2381 (2014).

20. X. Li, H. Wang, Y. Shimizu, A. Pyatenko, K. Kawaguchi, and N. Koshizaki, Chem. Commun. 47, 932 (2011).

21. T. N. Lin, K. H. Chih, C. T. Yuan, J. L. Shen, C. A. J. Lin, and W. R. Liu, Nanoscale 7, 2708 (2015).

22. P. J. F. Harris, Crit. Rev. Solid State Mater. Sci. 30, 235 (2005).

23. Y. Dong, C. Chen, X. Zheng, L. Gao, Z. Cui, H. Yang, C. Guo, Y. Chi, and C. M. Li, J. Mater. Chem. 22, 8764 (2012).

24. B. Han, M. Yu, T. Pen, Y. Li, X. Hu, R. Xiang, X. Hou, and G. He, New J. Chem. 41, 5267 (2017).

25. L. Li, G. Wu, G. Yang, J. Peng, J. Zhao, and J.-J. Zhu, Nanoscale 5, 4015 (2013).

26. M. Bacon, S. J. Bradley, and T. Nann, Part. Part. Syst. Charact. 31, 415 (2014).

27. A. B. Kuzmenko, E. van Heumen, F. Carbone, and D. van der Marel, Phys. Rev. Lett. 100, 117401 (2008).

28. S. Kang, K. M. Kim, K. Jung, Y. Son, S. Mhin, J. H. Ryu, K. B. Shim, B. Lee, H. Han, and T. Song, Sci. Rep. 9, 4101 (2019).

29. T. Fan, W. Zeng, W. Tang, C. Yuan, S. Tong, K. Cai, Y. Liu, W. Huang, Y. Min, and A. J. Epstein, Nanoscale Res. Lett. 10, 55 (2015).

30. S. Kang, Y. K. Jeong, J. H. Ryu, Y. Son, W. R. Kim, B. Lee, K. H. Jung, and K. M. Kim, Appl. Surf. Sci. 506, 144998 (2020).

31. G. Yang, Prog. Mater. Sci. 52, 648 (2007).

32. D. Zhang, B. Gokce, and S. Barcikowski, Chem. Rev. 117, 3990 (2017).

33. N. Takada, A. Fujikawa, N. Koshizaki, and K. Sasaki, Appl. Phys. A 110, 835 (2012).

34. S. He, M. J. Turnbull, Y. Nie, X. Sun, and Z. Ding, Surf. Sci. 676, 51 (2018). 
35. R. Zhang, J. R. Adsetts, Y. Nie, X. Sun, and Z. Ding, Carbon 129, 45 (2018).

36. K. Habiba V. I. Makarov, J. Avalos, M. J. F. Guinel, B. R. Weiner, and G. Morell, Carbon 64, 341 (2013)

37. D. Qu, M. Zheng, J. Li, Z. Xie, and Z. Sun, Light Sci. Appl. 4, e364 (2015).
38. F. Liu, M. H. Jang, H. D. Ha, J. H. Kim, Y. H. Cho, and T. S. Seo, Adv. Mater. 25, 3657 (2013).

39. S. Chen, N. Ullah, T. Wang, and R. Zhang, J. Mater. Chem. C 6, 6875 (2018).

40. X. Hai, J. Feng, X. Chen, and J. Wang, J. Mater. Chem. B 6, 3219 (2018). 